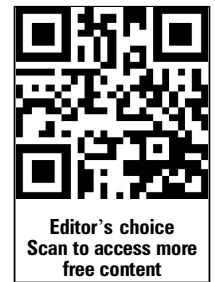

Division of Clinical Epidemiology and Aging Research, German Cancer Research Center (DKFZ), Heidelberg, Germany ${ }^{2}$ Klinik Schwabenland, Isny-Neutrauchburg, Germany

Correspondence to Dr Ute Mons, Division of Clinical Epidemiology and Aging Research, German Cancer Research Center (DKFZ), Im Neuenheimer Feld 581, 69120 Heidelberg, Germany; u.mons@dkfz.de

Received 12 November 2013 Revised 30 January 2014 Accepted 25 February 2014 Published Online First 14 May 2014

\section{SLinked}

- http://dx.doi.org/10.1136 heartjnl-2013-305304 - http://dx.doi.org/10.1136/ heartjnl-2014-305780

\section{CrossMark}

To cite: Mons $U$, Hahmann $\mathrm{H}$, Brenner $\mathrm{H}$. Heart 2014; 100: 1043-1049.

\title{
A reverse J-shaped association of leisure time physical activity with prognosis in patients with stable coronary heart disease: evidence from a large cohort with repeated measurements
}

\author{
Ute Mons, ${ }^{1}$ Harry Hahmann, ${ }^{2}$ Hermann Brenner ${ }^{1}$
}

\begin{abstract}
Objective To study the association of self-reported physical activity level with prognosis in a cohort of patients with coronary heart disease (CHD), with a special focus on the dose-response relationship with different levels of physical activity.

Methods Data were drawn from a prospective cohort of 1038 subjects with stable CHD in which frequency of strenuous leisure time physical activity was assessed repeatedly over 10 years of follow-up. Multiple Cox proportional hazards regression models were used to assess the association of physical activity level with different outcomes of prognosis (major cardiovascular events, cardiovascular mortality, all-cause mortality), with different sets of adjustments for potential confounders and taking into account time-dependence of frequency of physical activity.
\end{abstract}

Results A decline in engagement in physical activity over follow-up was observed. For all outcomes, the highest hazards were consistently found in the least active patient group, with a roughly twofold risk for major cardiovascular events and a roughly fourfold risk for both cardiovascular and all-cause mortality in comparison to the reference group of moderately frequent active patients. Furthermore, when taking time-dependence of physical activity into account, our data indicated reverse J-shaped associations of physical activity level with cardiovascular mortality, with the most frequently active patients also having increased hazards (2.36, 95\% Cl 1.05 to 5.34 ).

Conclusions This study substantiated previous findings on the increased risks for adverse outcomes in physically inactive CHD patients. In addition, we also found evidence of increased cardiovascular mortality in patients with daily strenuous physical activity, which warrants further investigation.

\section{INTRODUCTION}

Extensive research has established that physical activity is inversely associated with the risk of developing cardiovascular disease in healthy adults. ${ }^{1}$ In patients with manifest cardiovascular disease, current clinical practice guidelines recommend encouraging patients to undertake daily moderate intensity physical exercise for secondary prevention. For example, a pertinent US guideline recommends 30-60 min moderate intensity aerobic activity, such as brisk walking, at least 5 days and preferably 7 days per week. ${ }^{2}$
While such recommendations are based on numerous clinical trials clearly showing that exercise-based cardiac rehabilitation improves prognosis in heart disease patients, ${ }^{3}{ }^{4}$ only a few prospective studies have examined the potential benefit of physical activity in clinical practice under reallife conditions. These consistently found the highest risks for adverse outcomes in the most inactive patients, ${ }^{5-10}$ yet the exact dose-response relationship with different levels of physical activity is still unclear. For example, one study found linear trends towards lower mortality with increasing levels of exercise; ${ }^{8}$ another study found a curvilinear association, with the greatest relative mortality differences in those with lower physical activity level and a levelling off of the risk with increasing levels ${ }^{7}$; and another study even showed increased mortality rates in the vigorously active group. ${ }^{10}$

In some disagreement with the tenor of recommendations outlined in clinical practice guidelines, these studies consistently reported substantial benefits of physical activity already at lower levels compared to inactive subjects, but with higher levels not conferring much (if any) additional benefit. Of note, most studies measure or consider physical activity at baseline only, which may lead to an underestimation of the risk in the lowest activity group since physical activity level is likely to decrease with age and thus over time. ${ }^{11}$

In this study, we investigated the association of leisure time physical activity level with prognosis in a cohort of patients with coronary heart disease (CHD). We were especially interested in the doseresponse relationship with different levels of physical activity and also took changes in physical activity level during long-term follow-up into account.

\section{METHODS}

Study design and participants

Data from the KAROLA study were used for this investigation. As reported in detail elsewhere, ${ }^{12}$ the KAROLA study is a prospective cohort study comprising subjects that were recruited in 1999 and 2000 in two rehabilitation clinics in middle and southern Germany (Klinik am Südpark, Bad Nauheim; Schwabenland-Klinik, Isny-Neutrauchburg). All CHD patients aged 30-70, who were undergoing inpatient cardiovascular rehabilitation in these clinics because of the recent occurrence of an acute cardiovascular event or procedure (acute coronary 
syndrome, acute myocardial infarction, coronary artery revascularisation) within the past 3 months before admission, were eligible for the study. Overall, the study included 1206 patients at baseline.

The study protocol was approved by the ethics committees of the Universities of Ulm and Heidelberg, and by the ethics boards of the chambers of physicians of the federal states of Hessen and Baden-Wuerttemberg. Written informed consent was obtained from all participants before enrolment in the study.

\section{Baseline and follow-up examinations}

Health related and sociodemographic data were collected from patients with self-administered standardised questionnaires at rehabilitation. Additional information on medical findings and secondary diagnoses was obtained from hospital medical records. Patients were recontacted 1, 3, 4.5, 6, 8 and 10 years after discharge from the rehabilitation clinic to collect lifestyle related, health related and sociodemographic information by means of self-administered standardised questionnaires. In addition, primary care physicians of patients were contacted to obtain medical information and incidence of cardiovascular events. For patients deceased during follow-up, death certificates were retrieved from local health authorities and the main cause of death was coded according to the current International Classification of Diseases (ICD).

\section{Outcomes}

Three different outcomes of prognosis were examined over 10 years of follow-up in this study. Occurrence of major cardiovascular events is a combined endpoint that included both fatal and non-fatal cardiovascular events (myocardial infarction and stroke; ie, ICD-9, pos. 390-459 up to year 4.5 follow-up and ICD-10, pos. I00-I99 thereafter). These were defined as either cardiovascular disease as main cause of death (as stated in the death certificate) or non-fatal events as reported by the primary care physician. Cardiovascular mortality as endpoint included only deaths from cardiovascular disease (as stated in the death certificate), and all-cause mortality as endpoint included deaths of any cause.

\section{Assessment of physical activity}

The first measurement of physical activity level outside of the rehabilitation programme was with the patient questionnaire at the year 1 follow-up. Frequency of leisure time physical activity was assessed with the question 'On average, how often have you engaged in physically strenuous and sweat-inducing activity in your leisure time in the past 12 months (ie, cycling, speedy hiking, gardening, sport)?' Answers were categorised into 'daily', '5 to 6 times per week', ' 2 to 4 times per week', ' 1 to 4 times per month' and 'rarely or never'. Further assessments with the exact same question were conducted $1,3,6,8$ and 10 years after rehabilitation. In addition, patients were asked to give the overall duration of these activities as average number of hours per week at year 1 follow-up.

\section{Covariates}

Sociodemographic covariates of interest were age, sex, school education and employment status. Included health related measures were body mass index (BMI), smoking status, self-reported poor health, number of affected vessels, LV function, and history of myocardial infarction, stroke, diabetes mellitus or hypertension. BMI was categorised as normal $\left(<25 \mathrm{~kg} / \mathrm{m}^{2}\right)$, overweight $\left(\geq 25-30 \mathrm{~kg} / \mathrm{m}^{2}\right)$ and obese $\left(>30 \mathrm{~kg} / \mathrm{m}^{2}\right)$. Self-reported smoking status was categorised as current, former and never-smoking. In order to reduce potential misclassification bias due to misreporting, the smoking status at the year 1 follow-up was validated based on serum cotinine measurements as reported in more detail elsewhere. ${ }^{13}{ }^{14}$ Briefly, self-reported non-smokers with a serum cotinine level of $\geq 15 \mathrm{ng} / \mathrm{mL}$ were reclassified as smokers. Thus, 31 self-reported never-smokers, 108 self-reported former smokers and one subject with missing information were reclassified as smokers, which increased smoking prevalence at year 1 follow-up from 8.6 to $22.2 \%$. Subjective health status was assessed with the question 'How would you rate your current health status', with six response options ranging from 'very poor' to 'very good'. The responses 'very poor' and 'poor' were combined into a binary variable indicating self-reported poor health. The severity of CHD was categorised by the number of affected vessels based on angiographic evaluation (single-, double- and triple-vessel disease), according to the medical records at the rehabilitation clinic. Histories of myocardial infarction, stroke, diabetes mellitus and hypertension were constructed as binary variables, based on medical records at the rehabilitation clinic and physician reports at year 1 follow-up.

\section{Statistical analyses}

The study population was described with respect to various sociodemographic and medical characteristics. Crude relationships between physical activity level and outcomes of prognosis were examined by computation of incidence and mortality rates, respectively.

For the multivariate analyses, multiple Cox proportional hazards regression models were employed. For the categorical explanatory variable reflecting frequency of physical activity, the medium category was chosen as reference group (' 2 to 4 times per week'), since it represented the largest and a quite constant share of subjects over time and this approach further facilitated the examination of potential curvilinear relationships. The Cox models included adjustments for study site and for different sets of potential sociodemographic, lifestyle and health related potential confounders and predictors of prognosis (sex, age, education, employment status, smoking status, BMI, selfreported poor health, number of affected vessels, LV function, and history of myocardial infarction, diabetes or hypertension).

Since physical activity level is not constant over the life course, particularly in an elderly diseased population, but likely to decrease over time, an additional Cox model was fitted in which physical activity level was treated as a time-dependent predictor variable. ${ }^{15}$ In another model, other repeatedly measured covariates that are likely to change over time were introduced as time-varying covariates; these were BMI, self-reported poor health and employment status. Of note, these models exclude patients who dropped out over follow-up and did not participate in follow-up examinations (114 patients).

In order to explore dose-response relationships of duration of strenuous physical activity with outcomes of prognosis, restricted cubic splines analysis was employed. ${ }^{16}$

All statistical tests were two-sided, with an $\alpha$ level of 0.05 . SAS V.9.2 was used throughout.

\section{RESULTS}

\section{Cohort description}

In our cohort of patients with clinically manifest CHD, 1043 of 1188 subjects still alive at 1 year after rehabilitation (89\%) participated in the year 1 follow-up. Among these, 1038 subjects reported on the frequency of physical activity, constituting the study sample. The majority of subjects were older than 60 years (median age 61), male, overweight, current or former smoker, 
Table 1 Characteristics of the study sample at 1-year follow-up

\begin{tabular}{|c|c|}
\hline & $\mathrm{N}(\%)$ \\
\hline Total & $1038(100)$ \\
\hline \multicolumn{2}{|l|}{ Age } \\
\hline $30-49$ years & $151(14.6)$ \\
\hline $50-59$ years & $299(28.8)$ \\
\hline$\geq 60$ years & $588(56.7)$ \\
\hline \multicolumn{2}{|l|}{ Sex } \\
\hline Male & $884(85.2)$ \\
\hline Female & $154(14.8)$ \\
\hline \multicolumn{2}{|l|}{ Education } \\
\hline Low ( $\leq 9$ years) & $413(40.1)$ \\
\hline Moderate (10-11 years) & $235(22.8)$ \\
\hline High (12-13 years) & $178(17.3)$ \\
\hline Unknown & 7 \\
\hline \multicolumn{2}{|l|}{ Employment status } \\
\hline Employed & $315(30.4)$ \\
\hline Not employed, unemployed, retired & $722(69.6)$ \\
\hline Unknown & 1 \\
\hline \multicolumn{2}{|l|}{ BMI } \\
\hline$<25 \mathrm{~kg} / \mathrm{m}^{2}$ & $246(23.7)$ \\
\hline $25-30 \mathrm{~kg} / \mathrm{m}^{2}$ & $560(54.0)$ \\
\hline$>30 \mathrm{~kg} / \mathrm{m}^{2}$ & $231(22.3)$ \\
\hline Unknown & 1 \\
\hline \multicolumn{2}{|l|}{ Smoking status (cotinine-validated) } \\
\hline Never smoker & $307(29.7)$ \\
\hline Former smoker & $498(48.2)$ \\
\hline Current smoker & $229(22.2)$ \\
\hline Unknown & 4 \\
\hline \multicolumn{2}{|l|}{ Frequency of strenuous physical activity } \\
\hline Daily & $159(15.3)$ \\
\hline 5-6 times/week & $164(15.8)$ \\
\hline 2-4 times/week & $429(41.3)$ \\
\hline $1-4$ times/month & $192(18.5)$ \\
\hline Rarely/never & $94(9.1)$ \\
\hline \multicolumn{2}{|l|}{ Number of affected vessels } \\
\hline 1-vessel disease & $272(27.5)$ \\
\hline 2-vessel disease & $280(28.3)$ \\
\hline 3-vessel disease & $437(44.2)$ \\
\hline Unknown & 49 \\
\hline \multicolumn{2}{|l|}{ LV function } \\
\hline Not or only mildly impaired & $752(78.7)$ \\
\hline Moderately or severely impaired & $203(21.3)$ \\
\hline Unknown & 83 \\
\hline \multicolumn{2}{|l|}{ History of myocardial infarction } \\
\hline Yes & $602(58.0)$ \\
\hline No & $436(42.0)$ \\
\hline \multicolumn{2}{|l|}{ History of stroke } \\
\hline Yes & $44(4.6)$ \\
\hline No & $919(95.4)$ \\
\hline Unknown & 75 \\
\hline \multicolumn{2}{|l|}{ History of diabetes } \\
\hline Yes & $190(18.5)$ \\
\hline No & $839(81.5)$ \\
\hline Unknown & 9 \\
\hline \multicolumn{2}{|l|}{ History of hypertension } \\
\hline Yes & $698(68.0)$ \\
\hline No & $328(32.0)$ \\
\hline Unknown & 12 \\
\hline \multicolumn{2}{|l|}{ Self-reported health } \\
\hline Poor/very poor & $49(4.7)$ \\
\hline
\end{tabular}

Table 1 Continued

\begin{tabular}{lc}
\hline & $\mathbf{N}(\%)$ \\
\hline Not so good & $142(13.7)$ \\
Satisfactory & $473(45.7)$ \\
Good/very good & $372(35.9)$ \\
Unknown & 2 \\
Study centre & \\
$\quad$ Isny & $537(51.7)$ \\
Bad Nauheim & $501(48.3)$ \\
\hline
\end{tabular}

and had a history of myocardial infarction and hypertension (table 1). Around $40 \%$ of subjects were physically active 2 to 4 times per week, around $30 \%$ of subjects reported higher, and another $30 \%$ reported lower frequencies of physical activity, with around $10 \%$ reporting that they rarely or never engaged in physical activity. Median duration of strenuous activity was $5 \mathrm{~h}$ per week (IQR 7).

\section{Physical activity level over time}

We observed substantial changes of physical activity level over follow-up (figure 1). While the share of patients engaging in physical activity 5 to 6 times per week or daily decreased continuously over time, the share of those who were never physically active or for only 1 to 4 times per month increased. The portion of subjects who engaged in physical activity 2 to 4 times a week however remained comparably stable over time at around $40 \%$.

\section{Associations of physical activity level with prognosis}

Mean follow-up time $( \pm \mathrm{SD})$ was 8.1 years $( \pm 3.1)$.

The crude rates of incidence of major cardiovascular events and mortality rates were consistently highest in the least physically active group ('rarely/never') and lowest in those who engaged in physical activity 2 to 4 times per week (table 2 ). Increased rates were also found in daily active subjects. For the mortality outcomes, the crude relationships with physical activity levels revealed a reverse J-shaped association with decreasing levels of physical activity, that is, with increased mortality rates

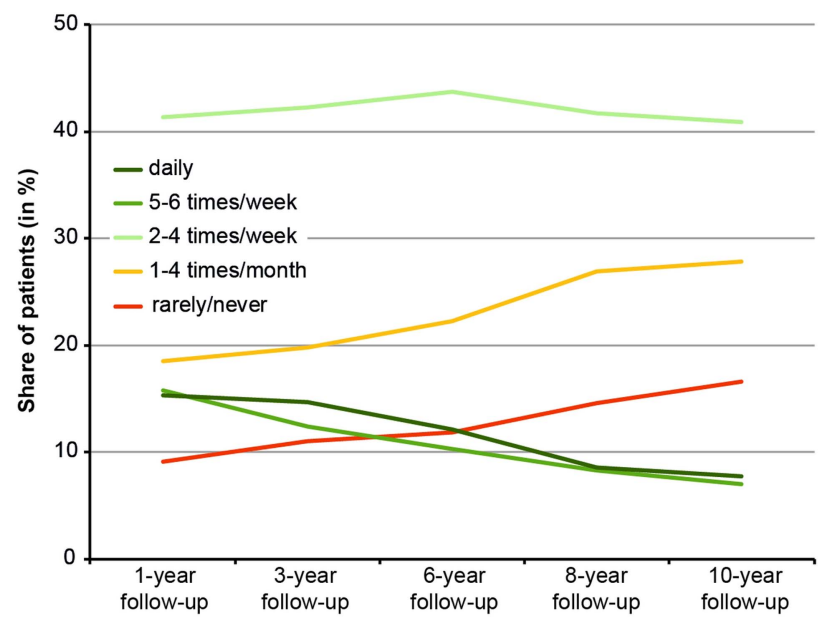

Figure 1 Distribution of physical activity level over follow-up. 
Table 2 Event numbers, person-years and crude event rates per 1000 person-years by frequency of strenuous physical activity at baseline

\begin{tabular}{|c|c|c|c|}
\hline & Events & Person-years & $\begin{array}{l}\text { Incidence/mortality rate } \\
(95 \% \mathrm{Cl} \text { ) per } 1000 \\
\text { person-years }\end{array}$ \\
\hline \multicolumn{4}{|c|}{ Major cardiovascular events } \\
\hline Daily & 24 & 1287.3 & 18.6 (12.5 to 27.8$)$ \\
\hline $5-6 \times /$ week & 29 & 1395.5 & 20.8 (14.4 to 29.9$)$ \\
\hline $2-4 \times /$ week & 61 & 3538.2 & 17.2 (13.4 to 22.2$)$ \\
\hline $1-4 \times /$ month & 39 & 1496.0 & 26.1 (19.1 to 35.7 ) \\
\hline Rarely/never & 29 & 624.5 & 46.4 (32.3 to 66.8$)$ \\
\hline \multicolumn{4}{|c|}{ Non-fatal cardiovascular events } \\
\hline Daily & 11 & 1211.7 & 9.1 (5.0 to 16.4$)$ \\
\hline $5-6 \times /$ week & 24 & 1351.3 & 17.8 (11.9 to 26.5$)$ \\
\hline $2-4 \times /$ week & 42 & 3382.6 & $12.4(9.2$ to 16.8$)$ \\
\hline $1-4 \times /$ month & 26 & 1372.1 & 19.0 (12.9 to 27.8$)$ \\
\hline Rarely/never & 9 & 476.0 & 18.9 (9.8 to 36.3$)$ \\
\hline \multicolumn{4}{|c|}{ Cardiovascular mortality } \\
\hline Daily & 14 & 1481.9 & 9.5 (5.6 to 16.0$)$ \\
\hline 5-6×/week & 10 & 1617.9 & 6.2 (3.3 to 11.5$)$ \\
\hline $2-4 \times /$ week & 19 & 4188.1 & 4.5 (2.9 to 7.1$)$ \\
\hline $1-4 \times /$ month & 15 & 1849.8 & 8.1 (4.9 to 13.5$)$ \\
\hline Rarely/never & 23 & 789.8 & 29.1 (19.4 to 43.8$)$ \\
\hline \multicolumn{4}{|c|}{ All-cause mortality } \\
\hline Daily & 24 & 1481.9 & 16.2 (10.9 to 24.2 ) \\
\hline $5-6 \times /$ week & 14 & 1617.9 & 8.7 (5.1 to 14.6$)$ \\
\hline $2-4 \times /$ week & 32 & 4188.1 & 7.6 (5.4 to 10.8$)$ \\
\hline $1-4 \times /$ month & 26 & 1849.8 & 14.1 (9.6 to 20.6$)$ \\
\hline Rarely/never & 35 & 789.8 & 44.3 (31.8 to 61.7$)$ \\
\hline
\end{tabular}

in both the most and the least frequently active, but with considerably higher mortality rates in the least physically active.

HRs for different levels of physical activity and outcomes of prognosis are reported in table 3. After adjustment for potential lifestyle and health related confounders, a roughly twofold increased risk for major cardiovascular events was observed in those who at the first measurement indicated rare or no physical activity (models 2 and 3), and persisted when treating physical activity and also other covariates as timedependent variables (models 4 and 5). In the models including time-dependent variables, a tendency towards increased risk was also observed for the group engaging in physical activity 1 to 4 times per month, and in those who engaged in daily physical activity.

For non-fatal cardiovascular events, no clear dose-response association was observed with different levels of physical activity.

The HRs for cardiovascular mortality were substantially and significantly increased in the least physically active group compared to the reference group of moderately active subjects in all models, with a roughly fourfold risk. The relationship tended to be reverse J-shaped, although a significantly increased HR in the most frequently active group was only observed in those models that included time-dependent covariates.

Comparable patterns were seen for physical activity and risk of all-cause mortality, with the HR being significantly increased and roughly four times higher in the least active group than in the reference group, and with a nearly twofold risk in the more frequently active groups. Again, the overall relationship tended to take a reverse J-shaped form.

Dose-response relationships of hours of physical activity per week with outcomes are shown in figure 2 . The dose-response curves reveal reverse J-shaped associations for cardiovascular and all-cause mortality.

\section{DISCUSSION}

In this observational study in more than 1000 patients with manifest CHD, we investigated the prognostic implications of self-reported leisure time physical activity. As expected, we observed evidence for a poorer prognosis in physically inactive patients. Furthermore, our data indicated a reverse J-shaped association of physical activity, especially with cardiovascular mortality: both inactive and daily active patients had increased hazards of mortality compared to the reference group of patients who were active 2 to 4 times per week, but with the hazards being highest in the inactive patient group.

Our data showed substantial decrease in engagement in physical activity over follow-up. The greatest and also a quite constant share of subjects were those who were moderately frequently active ( 2 to 4 times per week), which in our study was also the physical activity level that seemed to confer the greatest benefit in terms of survival. Pertinent current clinical practice guidelines however recommend physical activity on at least 5 days a week, ${ }^{2}{ }^{17}$ which in our study was accomplished only in less than one third of the baseline sample with decreasing tendency over time, and which was associated with increased hazards of cardiovascular mortality. One possible explanation for our study findings not being in agreement with recommendations of current guidelines could be that these are mainly based on evidence from randomised controlled trials. Such trials undoubtedly are the gold-standard method when evaluating the efficacy of interventions such as cardiovascular rehabilitation, but may have limited external validity with regard to applicability to real-life conditions because they apply strict inclusion and exclusion criteria that might lead to an exclusion of subjects at highest risk for adverse outcomes. Such issues have also been criticised by a pertinent meta-analysis on the effectiveness of cardiovascular rehabilitation, which found that the population studied in the included trials was predominantly male, middle aged and at low risk, and thus not representative of usual clinical practice. ${ }^{3}$

A potential explanation of our finding of worse prognosis in the most frequently physically active group could be that vigorous exercise increases the risk of ventricular arrhythmias and sudden cardiac death during or after exertion, especially in adults with heart conditions. ${ }^{18-21}$ While the risk of a cardiovascular event has been found to be low even during high-intensity exercise in a cardiovascular rehabilitation setting and especially with medical supervision, ${ }^{18}{ }^{22}$ the risk could however be increased with unsupervised leisure time exercise of comparable intensity. Unfortunately, our data do not allow identifying whether the recorded deaths occurred in relation to physical activity.

The repeated measurement of physical activity provided us with the opportunity to account for changes in physical activity level over time. The first assessment of physical activity was at the year 1 follow-up and referred to the past 12 months, that is, to the first year after cardiovascular rehabilitation. Since lifestyle modifications that are achieved during rehabilitation are often not maintained in the long run, ${ }^{23}$ it is not unlikely that the first measurement at 12 months post-rehabilitation did not accurately reflect true long-term physical activity patterns. Considering 
Table 3 Association of frequency of strenuous physical activity with outcomes of prognosis, results of Cox proportional hazards regression models

\begin{tabular}{|c|c|c|c|c|c|c|c|c|c|c|}
\hline & \multicolumn{2}{|c|}{ Model 1* } & \multicolumn{2}{|c|}{ Model $2 \dagger$} & \multicolumn{2}{|c|}{ Model 3‡ } & \multicolumn{2}{|c|}{ Model $4 \S$} & \multicolumn{2}{|c|}{ Model 5ף } \\
\hline & HR & $(95 \% \mathrm{Cl})$ & HR & $(95 \% \mathrm{Cl})$ & HR & $(95 \% \mathrm{Cl})$ & HR & $95 \%(\mathrm{Cl})$ & HR & $95 \%(\mathrm{Cl})$ \\
\hline \multicolumn{11}{|c|}{ Major cardiovascular events } \\
\hline Daily & 0.98 & (0.61 to 1.58 ) & 0.92 & (0.57 to 1.48$)$ & 1.03 & (0.61 to 1.73 ) & 1.50 & (0.85 to 2.66 ) & 1.54 & (0.87 to 2.71 ) \\
\hline 5-6x/week & 1.12 & (0.72 to 1.75$)$ & 1.11 & (0.71 to 1.73$)$ & 1.37 & (0.84 to 2.24 ) & 1.17 & (0.60 to 2.28$)$ & 1.16 & (0.60 to 2.25$)$ \\
\hline 2-4×/week & 1.00 & Ref. & 1.00 & Ref. & 1.00 & Ref. & 1.00 & Ref. & 1.00 & Ref. \\
\hline $1-4 \times /$ month & 1.46 & (0.98 to 2.20$)$ & 1.35 & (0.90 to 2.04$)$ & 1.43 & (0.90 to 2.27 ) & 1.55 & (0.95 to 2.54$)$ & 1.58 & (0.96 to 2.58$)$ \\
\hline Rarely/never & 2.77 & (1.75 to 4.37$)$ & 2.26 & (1.41 to 3.63$)$ & 2.03 & (1.21 to 3.42$)$ & 2.05 & (1.20 to 3.51$)$ & 1.85 & (1.06 to 3.23$)$ \\
\hline$N$ (Events) & \multicolumn{2}{|c|}{$1021(182)$} & \multicolumn{2}{|c|}{1015 (179) } & \multicolumn{2}{|c|}{$878(152)$} & \multicolumn{2}{|c|}{$881(123)$} & \multicolumn{2}{|c|}{$891(122)$} \\
\hline \multicolumn{11}{|c|}{ Non-fatal cardiovascular events } \\
\hline Daily & 0.68 & (0.35 to 1.32$)$ & 0.65 & (0.33 to 1.28$)$ & 0.71 & (0.33 to 1.52$)$ & 1.21 & (0.54 to 2.71$)$ & 1.20 & (0.54 to 2.69$)$ \\
\hline $5-6 \times /$ week & 1.37 & (0.83 to 2.28$)$ & 1.36 & (0.82 to 2.26 ) & 1.75 & (0.99 to 3.09 ) & 2.56 & (1.26 to 5.20$)$ & 2.52 & (1.24 to 5.12 ) \\
\hline $2-4 \times /$ week & 1.00 & Ref. & 1.00 & Ref. & 1.00 & Ref. & 1.00 & Ref. & 1.00 & Ref. \\
\hline $1-4 \times /$ month & 1.48 & (0.90 to 2.43 ) & 1.35 & (0.82 to 2.23 ) & 1.63 & (0.94 to 2.85 ) & 1.30 & (0.72 to 2.34 ) & 1.34 & (0.74 to 2.41 ) \\
\hline Rarely/never & 1.49 & (0.72 to 3.11$)$ & 1.35 & (0.64 to 2.83 ) & 1.37 & (0.62 to 3.01 ) & 2.51 & (1.38 to 4.56$)$ & 2.12 & (1.12 to 4.02 ) \\
\hline$N$ (Events) & \multicolumn{2}{|c|}{$951(112)$} & \multicolumn{2}{|c|}{947 (111) } & \multicolumn{2}{|c|}{$819(93)$} & \multicolumn{2}{|c|}{$806(91)$} & \multicolumn{2}{|c|}{$802(89)$} \\
\hline \multicolumn{11}{|c|}{ Cardiovascular mortality } \\
\hline Daily & 1.79 & (0.89 to 3.60$)$ & 1.65 & (0.82 to 3.32$)$ & 1.73 & (0.83 to 3.60$)$ & 2.36 & (1.05 to 5.34$)$ & 2.37 & (1.05 to 5.34$)$ \\
\hline $5-6 \times /$ week & 1.18 & (0.55 to 2.55 ) & 1.13 & (0.52 to 2.43 ) & 1.19 & (0.52 to 2.71 ) & 1.23 & (0.43 to 3.51$)$ & 1.22 & (0.43 to 3.46 ) \\
\hline $2-4 \times /$ week & 1.00 & Ref. & 1.00 & Ref. & 1.00 & Ref. & 1.00 & Ref. & 1.00 & Ref. \\
\hline $1-4 \times /$ month & 1.70 & (0.86 to 3.36 ) & 1.58 & (0.80 to 3.14 ) & 1.30 & (0.60 to 2.85 ) & 1.48 & (0.66 to 3.32 ) & 1.53 & (0.68 to 3.44 ) \\
\hline Rarely/never & 6.40 & (3.43 to 11.96 ) & 5.07 & (2.67 to 9.63 ) & 3.80 & (1.84 to 7.86 ) & 3.30 & (1.61 to 6.78$)$ & 3.39 & (1.62 to 7.10 ) \\
\hline$N$ (Events) & \multicolumn{2}{|c|}{$1030(81)$} & \multicolumn{2}{|c|}{$1024(79)$} & \multicolumn{2}{|c|}{$885(70)$} & \multicolumn{2}{|c|}{$889(65)$} & \multicolumn{2}{|c|}{$900(65)$} \\
\hline \multicolumn{11}{|c|}{ All-cause mortality } \\
\hline Daily & 1.78 & (1.04 to 3.04 ) & 1.64 & (0.96 to 2.81$)$ & 1.62 & (0.92 to 2.88 ) & 1.69 & (0.86 to 3.33 ) & 1.77 & (0.90 to 3.47$)$ \\
\hline $5-6 \times /$ week & 1.00 & (0.53 to 1.87 ) & 0.94 & (0.50 to 1.76$)$ & 1.00 & (0.52 to 1.96$)$ & 1.70 & (0.82 to 3.53 ) & 1.69 & (0.81 to 3.50 ) \\
\hline $2-4 \times /$ week & 1.00 & Ref. & 1.00 & Ref. & 1.00 & Ref. & 1.00 & Ref. & 1.00 & Ref. \\
\hline $1-4 \times /$ month & 1.75 & (1.04 to 2.95 ) & 1.60 & (0.95 to 2.70 ) & 1.53 & (0.86 to 2.72 ) & 1.69 & (0.92 to 3.10$)$ & 1.74 & (0.95 to 3.21 ) \\
\hline Rarely/never & 5.36 & (3.26 to 8.81 ) & 4.52 & (2.71 to 7.52 ) & 3.50 & (1.97 to 6.20$)$ & 3.92 & (2.26 to 6.79 ) & 3.81 & ( 2.17 to 6.70 ) \\
\hline$N$ (Events) & \multicolumn{2}{|c|}{$1030(130)$} & \multicolumn{2}{|c|}{$1024(128)$} & \multicolumn{2}{|c|}{885 (114) } & \multicolumn{2}{|c|}{$889(110)$} & \multicolumn{2}{|c|}{$900(110)$} \\
\hline
\end{tabular}

${ }^{*}$ Adjusted for sex, age, education and study site. Physical activity refers to year 1 follow-up measurement.

†As model 1, but additionally adjusted for employment status, cotinine-validated smoking status and BMI.

¥As model 2, but additionally adjusted for self-reported poor health, history of myocardial infarction, diabetes mellitus, hypertension, LV function and number of affected vessels.

$\S$ As model 3, but treating physical activity as time-dependent predictor variable.

ๆAs model 4, but additionally treating BMI, self-reported poor health and employment status as time-dependent covariates.

only baseline level of physical activity, as done in previous studies with one notable exception, ${ }^{6}$ could therefore lead to an underestimation of the risk in the lowest activity group as demonstrated by a methodological study. ${ }^{11}$ This is supported by our results, which revealed higher HRs for inactive patients in the model taking time-dependence into account compared to the baseline prediction model with an identical set of adjustments. In addition, the HRs in the most active patient group tended to be underestimated in the baseline prediction models. Of note, besides accounting for changes in physical activity over time, using a medium exposure group as the reference group could also have contributed to reducing potential bias, since physically inactive subjects are possibly more likely to also have other unfavourable health behaviours or to have poorer health.

A major limitation of this study is the relatively crude assessment of our explanatory variable, which was solely based on self-report. The measure was further limited by relying on subjects' definitions of strenuous and sweat-inducing physical activity and not assessing type and intensity of activities. However, despite some potential for misclassification, solely assessing the time dimension of strenuous physical activity in our cohort of mostly older subjects with manifest $\mathrm{CHD}$ and frequent co-morbid conditions, whose abilities to exercise might be generally limited, could still be sufficient for ranking subjects with regard to their level of activity. In addition, even though the HRs in the most active group were consistently above 1 for the mortality outcomes, the confidence limits often included 1 and our results might thus also be due to chance. Although we believe that the consistency and robustness of our results support the general validity of our findings, a more detailed instrument would have provided the opportunity for more in-depth and confirmatory analyses.

Because patients with poorer health are less likely to engage in strenuous physical activity, reverse causation bias could have led to an overestimation of the hazards in the inactive patient group and to an underestimation in the most physically active. However, we adjusted for baseline history of co-morbid conditions and for self-reported poor health in order to limit the potential for bias.

Physical activity seemed to be most strongly associated with mortality outcomes, but it should be kept in mind that the presented endpoints are not distinct, with fatal cardiovascular events being a subset of both major cardiovascular events and 

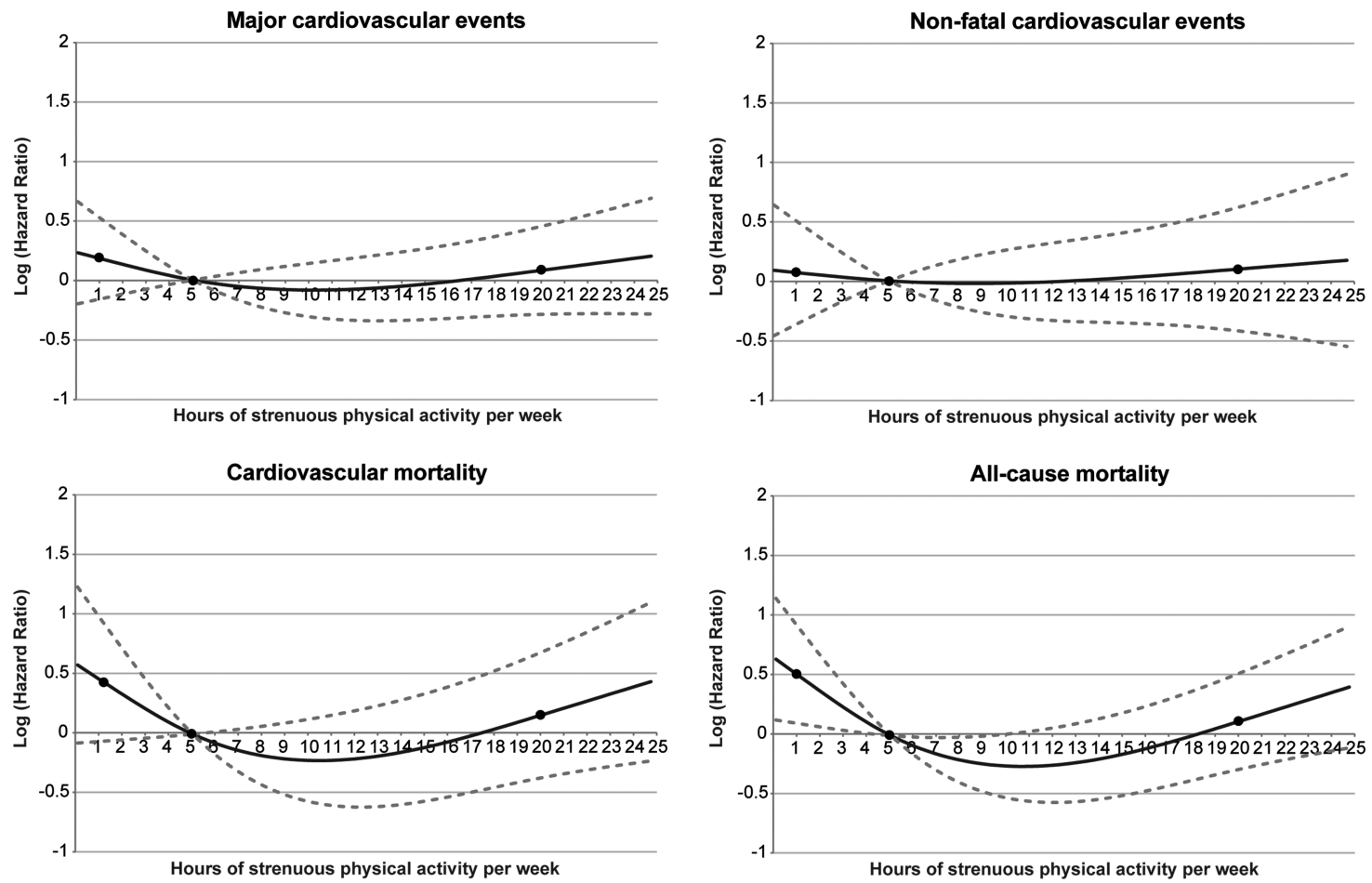

Figure 2 Relationship between overall duration of strenuous activity in hours per week and different outcomes of prognosis. Solid black line: point estimates; grey dashed lines: $95 \%$ Cls. The black circles in the point estimates curve represent the knots, which were set at the 5th, 50th and 95th percentiles according to the distributions of the continuous exposure variable. The median was set as the reference value. The models were adjusted for season, sex, age, education, employment status, study site, cotinine-validated smoking status, BMI, self-reported poor health, history of myocardial infarction, diabetes mellitus, hypertension and number of affected vessels.

all-cause mortality, which could partly explain similarities in observed associations.

Finally, although we carefully controlled for a number of potential confounders, we cannot rule out the possibility of residual confounding by imperfect measurement of characteristics or by unmeasured confounders.

To conclude, this study substantiated previous findings on the health benefits of physical activity in patients with manifest CHD: subjects who rarely or never engage in physical activity showed a substantially worse prognosis than those who were physically active for 2 to 4 times per week. Physical activity should thus be considered an integral part of a long-term secondary prevention strategy and further encouraged in inactive patients. In addition, consistent with the results of previous studies, despite differences in assessment of physical activity, ${ }^{7} 810$ we found that higher frequencies of physical activity did not confer additional benefit beyond that of physical activity of moderate frequency and duration, which suggests the existence of an upper limit for benefits. In some agreement with one previous study, ${ }^{10}$ our data even suggest that daily active subjects might have poorer prognosis compared to the moderately frequently active. Given the limitation of our physical activity assessment, further investigations of a potential reverse J-shaped association of physical activity with prognosis in CHD patients seem warranted. In order to inform recommendations on the optimal amount of physical activity for patients with cardiovascular disease, further research is particularly needed on whether there exists a threshold at which risks outweigh the benefits.

\section{Key messages}

What is already known on this subject

- It is established that physical activity improves prognosis in heart disease patients.

- Current clinical practice guidelines recommend up to daily moderate-intensity physical exercise in heart disease patients, although the dose-response relationship of physical activity with prognosis is still unclear.

What this study adds

- We examined the association of leisure time physical activity with prognosis in a prospective cohort study comprising patients with stable coronary heart disease.

- As expected, the highest hazards for adverse outcomes were found in the least active patient group.

- However, substantially increased hazards were also observed in the most frequently physically active groups, indicating reverse J-shaped associations of physical activity level with cardiovascular mortality.

Contributors UM carried out the statistical analyses and drafted the manuscript. $\mathrm{HB}$ is the principal investigator of the KAROLA study. All authors contributed to the interpretation of the data, revised drafts critically for important intellectual content, and reviewed and approved the final manuscript. 
Funding This work was supported by grants from the German Federal Ministry of Education and Research (01GD9820/0, 01ER0814), and the Willy Robert Pitzer Foundation, Bad Nauheim, Germany. The funders played no role in the design and conduct of the study; collection, management, analysis and interpretation of the data; and preparation, review, submission or approval of the manuscript.

\section{Competing interests None.}

Patient consent Obtained.

Ethics approval Ethics Committee of the University of Ulm; Ethics Committee of the University of Heidelberg; Ethics Board of the Chambers of Physicians of the federal state of Hessen; Ethics Board of the Chambers of Physicians of the federal state of Baden-Wuerttemberg.

Provenance and peer review Commissioned; externally peer reviewed.

\section{REFERENCES}

1 Li J, Siegrist J. Physical activity and risk of cardiovascular disease-a meta-analysis of prospective cohort studies. Int I Environ Res Public Health 2012;9:391-407.

2 Fihn SD, Gardin JM, Abrams J, et al. 2012 ACCF/AHA/ACP/AATS/PCNA/SCAI/STS guideline for the diagnosis and management of patients with stable ischemic heart disease: executive summary: a report of the American College of Cardiology Foundation/American Heart Association task force on practice guidelines, and the American College of Physicians, American Association for Thoracic Surgery, Preventive Cardiovascular Nurses Association, Society for Cardiovascular Angiography and Interventions, and Society of Thoracic Surgeons. Circulation 2012;126:3097-137.

3 Heran BS, Chen JM, Ebrahim S, et al. Exercise-based cardiac rehabilitation for coronary heart disease. Cochrane Database Syst Rev 2011:CD001800.

4 Lawler PR, Filion KB, Eisenberg MJ. Efficacy of exercise-based cardiac rehabilitation post-myocardial infarction: a systematic review and meta-analysis of randomized controlled trials. Am Heart J 2011;162:571-84, e2.

5 Apullan FJ, Bourassa MG, Tardif JC, et al. Usefulness of self-reported leisure-time physical activity to predict long-term survival in patients with coronary heart disease. Am J Cardiol 2008:102:375-9.

6 Gerber Y, Myers V, Goldbourt U, et al. Long-term trajectory of leisure time physical activity and survival after first myocardial infarction: a population-based cohort study. Eur J Epidemiol 2011;26:109-16.

7 Janssen I, Jolliffe CJ. Influence of physical activity on mortality in elderly with coronary artery disease. Med Sci Sports Exerc 2006;38:418-7.

8 Moholdt T, Wisloff U, Nilsen TI, et al. Physical activity and mortality in men and women with coronary heart disease: a prospective population-based cohort study in Norway (the HUNT study). Eur I Cardiovasc Prev Rehabil 2008;15:639-45.
9 Shibata Y, Hayasaka S, Yamada T, et al. Physical activity and risk of fatal or non-fatal cardiovascular disease among CVD survivors: the JMS cohort study. Circ J 2011:75:1368-72.

10 Wannamethee SG, Shaper AG, Walker M. Physical activity and mortality in older men with diagnosed coronary heart disease. Circulation 2000;102: 1358-63

11 Andersen LB. Relative risk of mortality in the physically inactive is underestimated because of real changes in exposure level during follow-up. Am J Epidemiol 2004;160:189-95.

12 Rothenbacher $\mathrm{D}$, Koenig W, Brenner H. Comparison of N-terminal pro-B-natriuretic peptide, $\mathrm{C}$-reactive protein, and creatinine clearance for prognosis in patients with known coronary heart disease. Arch Intern Med 2006;166:2455-60.

13 Breitling LP, Rothenbacher D, Vossen CY, et al. Validated smoking cessation and prognosis in patients with stable coronary heart disease. J Am Coll Cardiol 2011:58:196-7.

14 Twardella D, Kupper-Nybelen J, Rothenbacher D, et al. Short-term benefit of smoking cessation in patients with coronary heart disease: estimates based on self-reported smoking data and serum cotinine measurements. Eur Heart J 2004:25:2101-8

15 Fisher LD, Lin DY. Time-dependent covariates in the Cox proportional-hazards regression model. Annu Rev Public Health 1999;20:145-57.

16 Desquilbet L, Mariotti F. Dose-response analyses using restricted cubic spline functions in public health research. Stat Med 2010;29:1037-57.

17 Skinner JS, Cooper A, Feder GS. Secondary prevention for patients after a myocardial infarction: summary of NICE guidance. BMJ 2007;334: $1112-13$.

18 Thompson PD, Franklin BA, Balady GJ, et al. Exercise and acute cardiovascular events placing the risks into perspective: a scientific statement from the American Heart Association Council on Nutrition, Physical Activity, and Metabolism and the Council on Clinical Cardiology. Circulation 2007;115:2358-68.

19 Batty GD, Shipley MJ, Marmot M, et al. Leisure time physical activity and coronary heart disease mortality in men symptomatic or asymptomatic for ischaemia: evidence from the Whitehall study. J Public Health Med 2003;25:190-6.

20 Marijon E, Tafflet M, Celermajer DS, et al. Sports-related sudden death in the general population. Circulation 2011;124:672-81.

21 Mont L. Arrhythmias and sport practice. Heart 2010;96:398-405.

22 Rognmo O, Moholdt T, Bakken $\mathrm{H}$, et al. Cardiovascular risk of high- versus moderate-intensity aerobic exercise in coronary heart disease patients. Circulation 2012:126:1436-40.

23 Twardella D, Merx H, Hahmann $\mathrm{H}$, et al. Long term adherence to dietary recommendations after inpatient rehabilitation: prospective follow up study of patients with coronary heart disease. Heart 2006;92:635-40. 